\title{
Generalized Maximum Entropy
}

\author{
Peter Cheeseman ${ }^{*}$ and John Stutz ${ }^{\dagger}$ \\ "NICTA, Locked Bag.6016, University of New South Wales, Kensington, NSW, 1466, Australia \\ ${ }^{\dagger}$ MS 269-1, NASA-Ames Research Center, Moffet Field, CA
}

\begin{abstract}
.
A long standing mystery in using Maximum Entropy (MaxEnt) is how to deal with constraints whose values are uncertain. This situation arises when constraint values are estimated from data, because of finite sample sizes. One approach to this problem, advocated by E.T. Jaynes [1], is to ignore this uncertainty, and treat the empirically observed values as exact. We refer to this as the classic MaxEnt approach. Classic MaxEnt gives point probabilities (subject to the given constraints), rather than probability densities. We develop an alternative approach that assumes that the uncertain constraint values are represented by a probability density (e.g. a Gaussian), and this uncertainty yields a MaxEnt posterior probability density. That is, the classic MaxEnt point probabilities are regarded as a multidimensional function of the given constraint values, and uncertainty on these values is transmitted through the MaxEnt function to give uncertainty over the MaxEnt probabilities. We illustrate this approach by explicitly calculating the generalized MaxEnt density for a simple but common case, then show how this can be extended numerically to the general case. This paper expands the generalized MaxEnt concept introduced in a previous paper [3].
\end{abstract}

\section{INTRODUCTION}

A mystery in using Maximum Entropy (MaxEnt) inference in practice is: "Where do the constraints come from?". The normalization constraint (1) comes from the logical requirement that the sum of all probabilities must equal 1 , since some event $i$ out of a set of events must occur.

$$
\forall i, 0 \leq P_{i} \leq 1, \quad \sum_{i} P_{i}=1,
$$

However, other constraints, such as the mean value constraint discussed by Jaynes in [2] are just asserted, without specifying where they come from and how their constraint values are found. Two possible sources of constraints are:

1. By Definition: Such as the normalization constraint; or constraints derived from logical requirements, physical laws, or by assumption.

2. By Measurement: The value of these constraints is inherently uncertain.

In the first type of constraint, uncertainty associated with the constraint value does not arise. However, for constraints based on measurements, the observed value can only be known with a precision dictated by the sample size. The Classic MaxEnt (CME) approach has no obvious mechanism for taking this uncertainty into account. Jaynes was well aware of this problem, and attempted to address it in his paper [1], at the end of section $B$, where he offered three approaches. The first is to ignore it-just use the 
empirically determined constraint values as if they are exact. The second solution is to generalize the classic maxent approach to accommodate the constraint uncertainty-the approach we take in this paper, although Jaynes dismisses this approach as ad hoc. The third approach is to introduce constraint uncertainty by adding extra variance constraints. We show that the first and last approaches are untenable, while the second approach is dictated by the laws of probability.

\section{THE CLASSIC MAXENT SOLUTION (CME)}

The principle of Maximum Entropy (MaxEnt) is a method for using constraint information to find a set of point probability values, $\vec{P}$, that assumes the least (Shannon) information consistent with the given constraints. When the given (linear) constraints are insufficient to uniquely constrain $\vec{P}$ to particular point values, MaxEnt picks out the unique distribution that satisfies all the constraints and also maximizes the entropy.

In the case of a finite set of $I$ mutually exclusive and exhaustive events, described by discrete probabilities $P_{i}$, the entropy is defined as:

$$
\mathrm{H}(\vec{P})=-\sum_{i=1}^{i=I} P_{i} \times \log P_{i}
$$

Given a set of $J$ independent linear constraints, including the normalization (1), each of the form:

$$
\overrightarrow{A_{j}} \cdot \vec{P}=C_{j}
$$

with $J<I$, the maximum entropy distribution may be found by the following procedure [2]: define the partition function:

$$
\mathrm{Z}(\vec{\lambda}) \equiv \sum_{i=1}^{I} \exp \left(-\sum_{j=1}^{J} \lambda_{j} A_{j i}\right)
$$

with the Lagrange multipliers $\vec{\lambda}$ determined by the set of $J$ simultaneous equations:

$$
\frac{\partial}{\partial \lambda_{j}} \log (Z(\vec{\lambda}))+C_{j}=0
$$

Then

$$
\mathrm{H}_{\max }=\log (\mathrm{Z}(\vec{\lambda}))+\vec{\lambda} \cdot \vec{C}
$$

and the corresponding probability distribution is:

$$
P_{i}=\mathrm{Z}(\vec{\lambda})^{-1} \exp \left(-\sum_{j=1}^{J} \lambda_{j} A_{j i}\right)=\mathrm{Z}(\vec{\lambda})^{-1} \prod_{=1}^{J} j \exp \left(-\lambda_{j} A_{j i}\right)
$$

Explicit solutions for the dice case are given below. These CME values are different from the Maximum A Posterior (MAP), Maximum Likelihood (ML) and Posterior Mean probability estimators, reflecting the different assumptions built-in to each estimator (see [3] for a discussion of these assumptions). 


\section{GENERALIZED MAXENT}

Equations (2)-(7) show that the maximum entropy point probabilities $P_{i}$ are a function of the constraint values $C_{j}$. If these $C_{j}$ s are estimated from a sample, then Bayes implies that our knowledge of their values is approximate and can be expressed as pdfs (e.g., Gaussians), whose width deceases with increasing sample size. Using the Jacobian determinant of the function relating the maxent $P_{i}$ s to the $C_{j}$ s, the joint posterior pdf on the $C_{j}$ s can in principle be transformed into a joint pdf on the $P_{i}$ s through the maximum Entropy constraint/function. We illustrate this process by a simple example using a threefaced dice with an experimentally determined mean value. For the three face dice, there are three unknown probabilities $\vec{P}=P_{1}, P_{2}, P_{3}$, one for each of the three faces. These probabilities must satisfy the following linear constraints (i.e, the normalization and mean values constraints):

$$
P_{1}+P_{2}+P_{3}=1, \quad \sum_{i=1}^{i=3} i P_{i}=P_{1}+2 P_{2}+3 P_{3}=\mu,
$$

where the value $\mu$ is only known approximately from data. Since all face values are either 1,2 or $3, \mu$ must be in the range 1 to 3 . Here, the set of linear constraint equations (3) reduces to equations (8). In this simple case there is only one degree of freedom left, and this is removed when the additional maxent constraint (2) is also imposed. Using (7), the resulting $P_{i}$ s are:

$$
P_{i}=\exp (-\kappa-i \lambda)
$$

where $\kappa$ is fixed by the normalization constraint and $\lambda$ is fixed by the mean value constraint. Let $x=\exp (-\lambda)$ and $z=\exp (-\kappa)$. Substituting into (8), we have (after eliminating $z$ ):

$$
x^{2}(\mu-3)+x(\mu-2)+\mu-1=0,
$$

whose only positive solution is:

$$
x(\mu)=\frac{\mu-2+\sqrt{-3 \mu^{2}+12 \mu-8}}{2(3-\mu)} .
$$

The normalizing constant (or partition function) $z(\mu)$ is found by substituting $x(\mu)$ from (ii) into the normalizing equation to give:

$$
z(\mu)=\frac{2(3-\mu)^{3}}{-3 \mu^{2}+\mu\left(\sqrt{-3 \mu^{2}+12 \mu-8}-14\right)+4 \sqrt{-3 \mu^{2}+12 \mu-8}-14} .
$$

Putting these equations together gives the desire maxent probabilities:

$$
P_{i}(\mu)=z(\mu) x(\mu)^{i},
$$

which in turn reduce to:

$$
P_{1}(\mu)=\frac{2(3-\mu)^{2}}{10-3 \mu+\sqrt{-3 \mu^{2}+12 \mu-8}}
$$




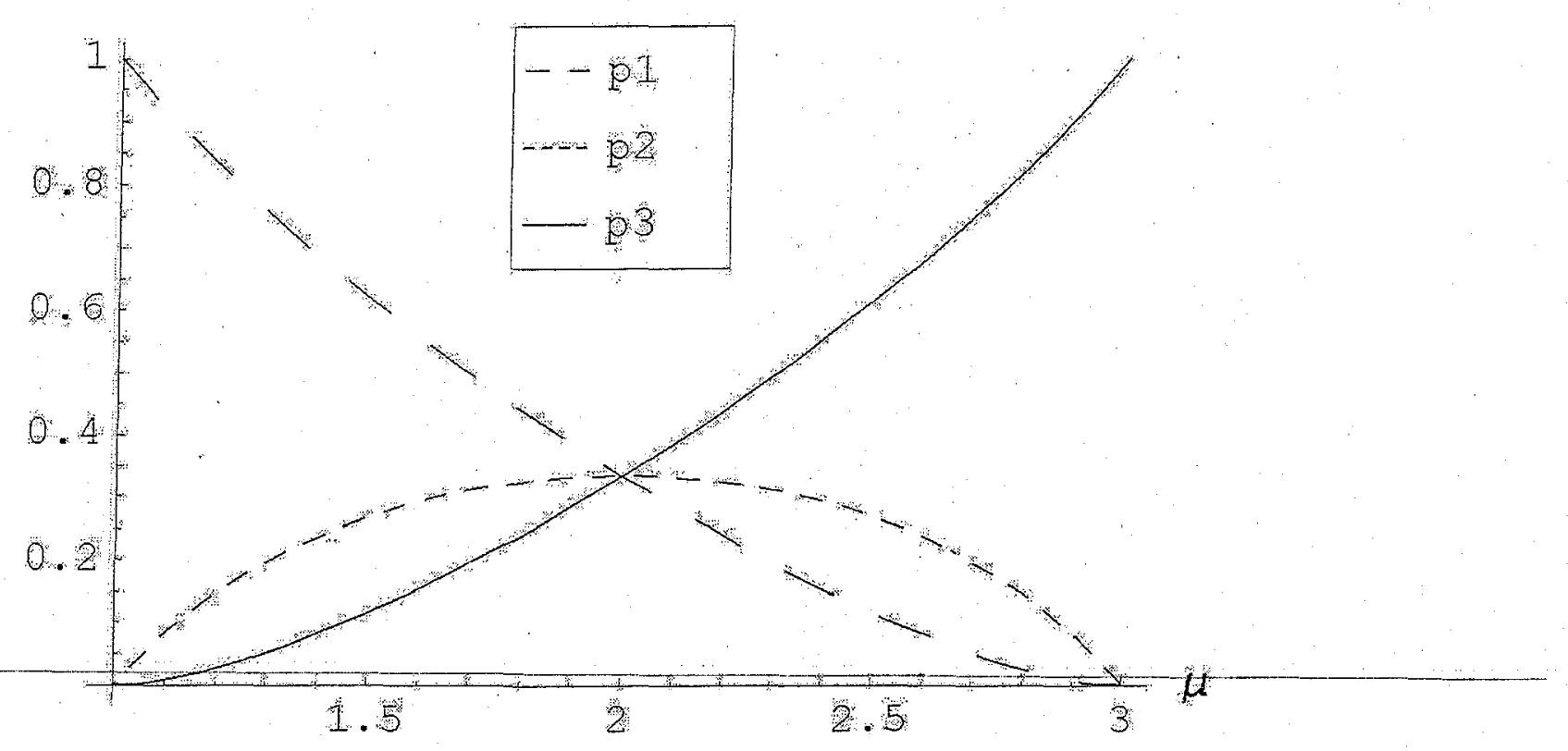

FIGURE 1. The maxent probabilities for each of the 3 faces as a function of $\mu$.

$$
\begin{aligned}
& P_{2}(\mu)=\frac{(3-\mu)\left(\mu-2+\sqrt{-3 \mu^{2}+12 \mu-8}\right)}{10-3 \mu+\sqrt{-3 \mu^{2}+12 \mu-8}} \\
& P_{3}(\mu)=\frac{\left(\mu-2+\sqrt{-3 \mu^{2}+12 \mu-8}\right)^{2}}{10-3 \mu+\sqrt{-3 \mu^{2}+12 \mu-8}} .
\end{aligned}
$$

This is the maxent solution to the three-faced dice problem as a function of $\mu$, and the resulting probabilities are shown in Fig. (1). Note that for $\mu=2, P_{i}=1 / 3$ for all $i$, as expected, since $\mu=2$ is the mean value for a "fair" dice, where all three faces are equally probable.

The maxent probabilities (14) can now be substituted into the entropy formula (2) to give the three faced dice entropy as a function of $\mu$, as shown in Fig. (2). Note that the entropy has a maximum at $\mu=2$, as expected, since this is the equiprobable entropy.

Having found the maxent point probabilities $\vec{P}$ as a function of $\mu$ for the 3 -faced dice problem, we now examine what happens if we do not know the value of $\mu$ exactly, but instead our knowledge is summarized as a pdf over $\mu$-i.e. $f_{\mu}(\mu)$. From standard probability theory, a pdf on one set of variables can be transformed into a pdf on an equal number of variables that are relat ed through a set of equations. That is:

$$
f_{y}\left(y_{1}, y_{2}, \cdots, y_{n}\right)=|D| f_{x}\left(x_{1}, x_{2}, \cdots, x_{n}\right),
$$


where $|D|$ is the Jacobian determinant of the transformation of the variables $x_{1}, x_{2}, \cdots, x_{n}$ to the variables $y_{1}, y_{2}, \cdots, y_{n}$, i.e.

$$
|D|=\frac{\partial\left(x_{1}, x_{2}, \cdots, x_{n}\right)}{\partial\left(y_{1}, y_{2}, \cdots, y_{n}\right)}=\left|\begin{array}{cccc}
\frac{\partial x_{1}}{\partial y_{1}} & \frac{\partial x_{1}}{\partial y_{2}} & \cdots & \frac{\partial x_{1}}{\partial y_{n}} \\
\frac{\partial x_{2}}{\partial y_{1}} & \frac{\partial x_{2}}{\partial y_{2}} & \cdots & \frac{\partial x_{2}}{\partial y_{n}} \\
\dot{x_{n}} & \frac{\partial x_{n}}{\partial y_{1}} & \cdots & \frac{\partial x_{n}}{\partial y_{n}}
\end{array}\right|
$$

For the three faced dice case, this is a one dimensional transformation for each $P_{i}$. For example, for the probability function $P_{2}(\mu),\left|D_{2}\right|$ is derived from (14) giving:

$$
\left|D_{2}\right|=\left|\frac{\partial \mu}{\partial P_{2}}\right|=\left|\frac{\sqrt{-3 \mu^{2}+12 \mu-8}}{2-\mu}\right|
$$

and similarly for $P_{1}$ and $P_{3}$. Note that (17) has a singularity at $\mu=2$, and that the absolute. value must be taken so that $\left|D_{2}\right|$ is always positive. Combining (15) and (17), we get finally:

$$
f_{P_{2}}\left(P_{2}\right)=\left|D_{2}\right| f_{\mu}(\mu)=\left|\frac{\sqrt{-3 \mu^{2}+12 \mu-8}}{2-\mu}\right| f_{\mu}(\mu),
$$

which has the desired effect of mapping the uncertainty about $\mu$ onto uncertainty about $P_{2}$. The singularity in (17) occurs at the maximum value for $P_{2}=1 / 3$, as can be seen in Fig. 1 , but the resulting pdf $f_{P_{2}}\left(P_{2}\right)$ is still normalized, despite the infinite value at this boundary. In the extreme case where $f_{\mu}(\mu)$ becomes a delta function, the corresponding $f\left(P_{i}\right)$ s also become delta functions, and give the CME result.

For the above simple three faced dice case, it was possible to do the analysis explicitly. Higher order cases cannot be done explicitly because they involve analytic roots of high order polynomials. In such cases, it is relatively easy to approximate the Jacobian determinant with a Taylor expansion about the estimated constraint values. In the simplest case, this involves the linear/tangent plane approximation of the maxent probability functions as the constraint values are numerically varied around their maximum values. This should be a good approximation provided the maxent probability functions do not curve significantly over the range of constraint values. In the three faced die case, Fig. (1), the $P_{i}$ s can be seen to be approximately straight lines for small $\mu$ ranges, showing that the linear approximation would work well in this case.

\section{DISCUSSION}

We call this mapping of uncertainty in the constraint values into uncertainty in the MaxEnt probabilities (expressed as pdfs) the Generalized principle of Maximum Entropy (GME). We have not previously seen this generalization in the literature, but it is a direct result of applying probability theory to the situation. In the limit of sample size $N \rightarrow \infty$, the constraint values are given exactly, so GME becomes CME. Jaynes in [1] end of section B, briefly mentions an approach that resembles GME, but he calls this approach ad hoc and does not elaborate. Instead he advocates adding the uncertainty on 


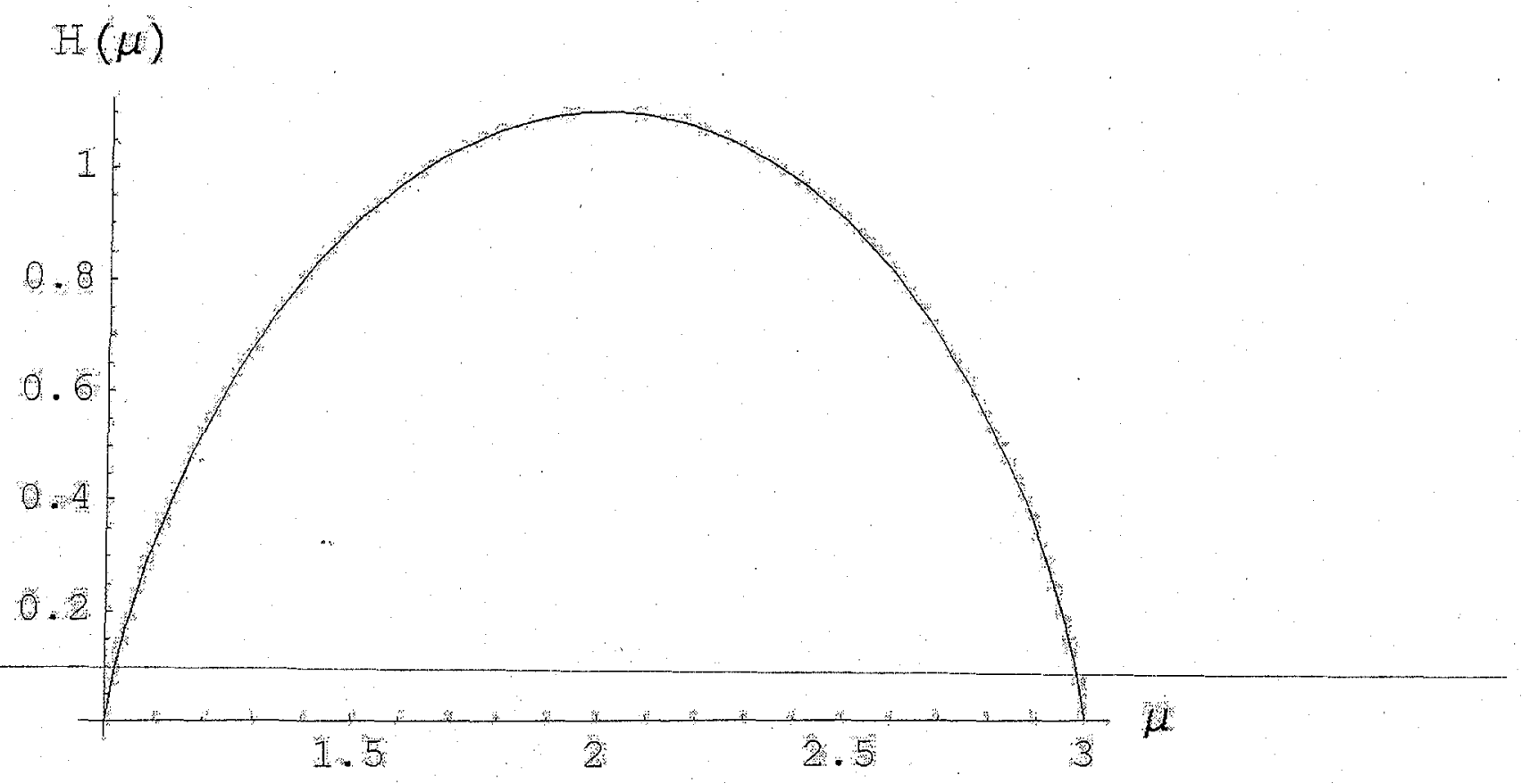

FIGURE 2. The entropy for the 3-faces die example as a function of $\mu$.

constraint values as extra constraints, such as a variance (on the constraint values). In our three faced dice problem, this would require adding asserting a variance on $\mu$. Jaynes did not develop this alternative approach, but if he had he would have discovered that it does not work, because the additional constraint(s) are on the wrong space! In CME, the constraints are on the space of possible probability values, $P_{i}$, not on the values of the constraints (such as $\mu$ ), so his extra constraints would not have any direct effect on the $P_{i}$ s. Even if it was possible to translate constraints on constraint values into constraints on the underlying $P_{i} \mathrm{~s}$, the resulting $\mathrm{CME}$ probabilities would be point probabilities, not pdfs, and so would not reflect the underlying uncertainty.

We stress that GME is only a solution to the problem of how to handle uncertain constraint values within the maximum entropy inference framework. The resulting pdfs avoid overly strong commitment compared with point probabilities. However, if the GME pdfs are used for prediction or decision making, it is important to remember that they embody other strong assumptions that may be incorrect in particular cases. The essential assumption is that the set of constraints used in either GME or CME is complete, meaning that no other significant constraints apply. Without good reason to believe in the constraint set's completeness, there is no good reason for believing the predictions of GME or CME. See [3] for further discussion on this point. 


\section{REFERENCES}

1. Jaynes, E. T., "Where do We Stand on Maximum Entropy", in The Maximum Entropy Formalism, edited by R. D. Levine and M. Tribus, MTT Press, Cambridge, MA, USA, 1978, pp. 15-118, http://bayes.wustl.edu/.

2. Jaynes, E. T., "Concentration of Distributions at Entropy Maxima", in E. T. Jaynes: Papers on Probability, Statistics, and Statistical Physics, edited by R. D. Rosenkrantz, D. Reidel, Dördrecht, 1983, chap. 11, pp. 315-336, http://bayes. wustl.edu/.

3. Cheeseman, P. C., and Stutz, J. C., "On the Relationship between Bayesian and Maximum Entropy Inference", in Bayesian Inference and Maximum Entropy Methods in Science and Engineering, edited by R. Fischer, R. Preuss, and U. von Toussaint, American Insititute of Physics, Melville, NY, USA, 2004, pp. 445-461. 